\title{
Diagnóstico de larvas de primeiro estágio de nematóides gastrintestinais de bezerros leiteiros do município de Paty do Alferes- RJ
}

\section{Diagnosis of first stage larvae of gastrointestinal nematodes of dairy calves in the city of Paty do Alferes-RJ}

\author{
Marcelo Abidu-Figueiredo ${ }^{1 *}$; Marcus Sandes Pires; \\ Argemiro Sanavria ${ }^{3}$; Richard Karl Reinecke ${ }^{4}$
}

\section{Resumo}

\begin{abstract}
Foi realizado um levantamento epidemiológico utilizando-se a técnica coproparasitologica do filtro de Visser para o diagnóstico de larvas de primeiro estágio $\left(\mathrm{L}_{1}\right)$ de nematóides gastrintestinais em amostras fecais de 150 bezerros leiteiros do município de Paty do Alferes, estado do Rio de Janeiro. Amostras individuais de fezes foram coletadas diretamente da ampola retal de 150 bezerros, com idade variando entre três e 12 meses, procedentes de 15 propriedades rurais no município de Paty do Alferes-RJ. Destas amostras, foram identificados os seguintes gêneros de nematóides: Haemonchus spp., Trichostrongylus spp., Cooperia spp., Bunostomum spp., Strongyloides e Oesophagostomum spp. Os gêneros de maior ocorrência foram Cooperia spp. (54\%), Haemonchus spp. (50,6\%) e Trichostrongylus spp. (48,7\%). Do total de propriedades vistoriadas, $86,7 \%$ apresentaram animais positivos para Haemonchus spp. e Trichostrongylus spp. O gênero Bunostomum spp. foi o que apresentou menor ocorrência, com 12,7\% de casos. Os gêneros identificados com os maiores valores médios de larvas de primeiro estágio em $15 \mathrm{~mL}$ de fezes por amostra foram: Cooperia spp. (812) e Haemonchus spp. (489).
\end{abstract}

Palavra-chave: Nematóides, bezerros leiteiros, larvas de primeiro estágio

\begin{abstract}
An epidemiologycal survey were carried out using the filter of Visser technique for the diagnosis of first-stage larvae $\left(\mathrm{L}_{1}\right)$ of gastrointestinal nematodes in faecal samples from 150 dairy calves in Paty do Alferes city, Rio de Janeiro state. Feces were individually collected directly of the rectal flask of 150 calves, with age varying between three and 12 months, coming from 15 rural properties in the municipal district Paty do Alferes-RJ. The following genera of nematodes were identified: Haemonchus spp, Trichostrongylus spp., Cooperia spp., Bunostomum spp., Strongyloides and Oesophagostomum spp. The genera of greater occurrence were Cooperia spp. (54\%), Haemonchus spp. (50.6\%) and Trichostrongylus spp. (48.7\%). Of the total of inspected properties, $86.7 \%$ presented positive animals for Haemonchus spp. and Trichostrongylus spp. The genera Bunostomum spp. had presented smaller occurrence, with $12.7 \%$ of cases. The genera identified with the highest medium values of first-stage larvae in $15 \mathrm{~mL}$ of faeces were: Cooperia spp. (812) and Haemonchus spp. (489).
\end{abstract}

Key words: Nematodes, dairy calves, first stage larvae

1 Prof. do Departamento de Biologia Animal, Instituto de Biologia, Universidade Federal Rural do Rio de Janeiro. Seropédica, RJ. E-mail: marceloabidu@gmail.com

2 Aluno do Curso de Pós-graduação em Ciências Veterinárias, Instituto de Veterinária, Universidade Federal Rural do Rio de Janeiro, CEP:23890-000, Seropédica, RJ. E-mail: marcussandes@yahoo.com.br

3 Prof. do Departamento de Epidemiologia e Saúde Pública, Instituto de Veterinária, Universidade Federal Rural do Rio de Janeiro, CEP: 23890-000, Seropédica, RJ. E-mail: sanavria@ufrrj.br

4 Prof. Emérito da Universidade de Pretória- África do Sul.

* Autor para correspondência 
Nos ruminantes, as infecções por helmintos gastrintestinais determinam perdas econômicas tanto pela mortalidade, quanto pela queda na produtividade dos animais (AMARANTE, 2004). O maior impacto econômico no sistema de criação esta relacionado aos casos de infecções subclínicas presentes no rebanho (FORBES; CUTLER; RICE, 2002). Particularmente, em bezerros, vários estudos demonstraram que as infecções por helmintos gastrintestinais afetam principalmente o crescimento do animal, ganho de peso e a conversão alimentar (LIMA, 1981).

Além disso, estudos regionalizados com o objetivo de se conhecer melhor as helmintoses mais importantes e frequentes na pecuária bovina vêm sendo desenvolvidos em todo o país, desde a década de setenta, permitindo conhecer melhor as características específicas para cada região (BIANCHIN, 1991).

A avaliação do grau de infecção animal, assim como também da contaminação ambiental, por meio de exames clínicos e laboratoriais são medidas importantes para o desenvolvimento de programas de controle eficientes (BIANCHIN et al., 1993; WALLER, 1999; EYSKER; PLOEGER, 2000). Em relação às técnicas de diagnostico coproparasitologicos, Abidu-Figueredo et al. (2005) observaram que a técnica utilizando o filtro de Visser (REINECKE; FIGUEIREDO; MATTOS JUNIOR, 1992) apresentou melhores resultados na detecção de ovos de nematóides gastrintestinais em búfalos.

O objetivo deste trabalho foi realizar um levantamento coproparasitologico utilizando o filtro de Visser (REINECKE; FIGUEIREDO; MATTOS JUNIOR, 1992), para a obtenção de larvas de primeiro estágio $\left(\mathrm{L}_{1}\right)$ de nematóides em bezerros leiteiros naturalmente infectados, localizados no município de Paty do Alferes - RJ.

Amostras de fezes foram coletadas, individualmente, diretamente da ampola retal de 150 bezerros, com idade variando entre três e 12 meses, procedentes de 15 propriedades rurais no município de Paty do Alferes, sendo coletado em cada propriedade, 10 amostras de forma aleatória. O município esta situado no estado do Rio de Janeiro, a $119 \mathrm{~km}$ da capital $\left(22^{\circ} 25^{\prime} 44^{\prime \prime}\right.$ latitude Sul e $43^{\circ} 25^{\prime} 8^{\prime \prime}$ longitude oeste, tendo $610 \mathrm{~m}$ de altitude).

As fezes dos animais foram acondicionadas em sacos plásticos, identificadas e colocadas em caixas de isopor com gelo, e encaminhadas ao Laboratório de Doenças Parasitárias do Convênio UFRRJ - EMBRAPA - Sanidade Animal localizado no município de Seropédica - RJ.

Os exames laboratoriais foram realizados de acordo com a técnica de Reinecke, Figueiredo e Mattos Junior et al. (1992), Reinecke e Fonseca (1992) e Reinecke e Reinecke (1993) no mesmo dia de chegada do material ao laboratório. O procedimento da técnica de diagnóstico adotada consiste na obtenção de larvas de primeiro estágio $\left(\mathrm{L}_{1}\right)$, a partir do processamento de $15 \mathrm{ml}$ de fezes de cada amostra, através do filtro de Visser, obtendo-se os resultados dos gêneros de nematóides em até 24 horas depois de iniciado o exame.

Os dados de ocorrência de parasitismo por nematóides em bezerros leiteiros em função do gênero do parasito observado no exame foram comparados, utilizando-se o teste não-paramétrico, Qui-quadrado $\left(\chi^{2}\right)$ a $5 \%$ de significância. As análises estatísticas foram realizadas utilizando o programa de análises estatísticas Biostat 2.0 (AYRES, 2000).

Os resultados obtidos a partir dos exames de fezes realizados com bezerros das propriedades do município de Paty do Alferes - RJ estão demonstrados na Tabela 1.

Dentre as 15 propriedades visitadas no município, 86,7\% apresentaram ocorrência de bezerros parasitados por Haemonchus spp. e Trichostrongylus spp., sendo também 73,3\% com animais positivos para Cooperia spp. e Oesophagostomum spp, e 40\% parasitados por Strongyloides e Bunostomum spp.

Os gêneros de nematóides que apresentaram maior ocorrência, dentre os 150 bezerros 
diagnosticados foram Cooperia spp. (54,0\%), (Tabela 1). Apesar do gênero Bunostomum spp. seguido de Haemonchus spp. (50,6\%) e apresentar menor ocorrência, perfazendo 12,7\% de Trychostrongylus spp. (48,7\%), não apresentando, casos, não houve diferença significativa $(\mathrm{p}>0,05)$ porém, diferença significativa $(\mathrm{p}>0,05)$ entre eles entre os casos positivos de Strongyloides $(21,6 \%)$.

Tabela 1. Número total e percentual de bezerros parasitados, assim como número total e percentual de propriedades com animais positivos aos diferentes gêneros de nematóides, identificados no município de Paty do Alferes-RJ.

\begin{tabular}{|c|c|c|c|c|}
\hline \multirow{3}{*}{ Gêneros identificados } & \multicolumn{2}{|c|}{ Bezerros $(n=150)$} & \multicolumn{2}{|c|}{ Propriedades $(n=15)$} \\
\hline & \multicolumn{2}{|c|}{ Positivos } & \multicolumn{2}{|c|}{ Positivos } \\
\hline & $\left(n^{0}\right)$ & $(\%)$ & $\left(n^{0}\right)$ & $(\%)$ \\
\hline Cooperia spp. & 81 & $54,0^{\mathrm{a}}$ & 11 & 73,3 \\
\hline Haemonchus spp. & 76 & $50,6^{\mathrm{a}}$ & 13 & 86,7 \\
\hline Trichostrongylus spp. & 73 & $48,7^{\mathrm{a}}$ & 13 & 86,7 \\
\hline Oesophagostomum spp. & 51 & $34,0^{\mathrm{b}}$ & 11 & 73,3 \\
\hline Strongyloides & 32 & $21,6^{c}$ & 6 & 40,0 \\
\hline Bunostomum spp. & 19 & $12,7^{\mathrm{c}}$ & 6 & 40,0 \\
\hline
\end{tabular}

Valores na coluna seguidos da mesma letra não diferem significativamente $(\mathrm{p}<0,05)$ pelo teste Qui-quadrado.

Em relação à média de larvas de primeiro estágio em $15 \mathrm{~mL}$ de fezes avaliada em cada amostra, dos bezerros examinados, observou-se que: os gêneros
Cooperia spp. (812) e Haemonchus spp. (489) apresentaram os maiores valores médios, sendo o gênero Bunostomum spp. (39), o que apresentou menor média ao exame laboratorial (Tabela 2).

Tabela 2. Valores médios de larvas de primeiro estágio $\left(\mathrm{L}_{1}\right)$ dos diferentes gêneros de nematóides observados, em 15 $\mathrm{mL}$ de fezes oriundos de bezerros naturalmente infectados localizados no município de Paty de Alferes, RJ.

\begin{tabular}{lc}
\hline Gêneros identificados & Média de $\mathrm{L}_{1} .15 \mathrm{~mL}^{-1}$ fezes $(\mathrm{n}=150)$ \\
\hline Haemonchus spp. & 489 \\
Trichostrongylus spp. & 216 \\
Cooperia spp. & 812 \\
Bunostomum spp. & 39 \\
Strongyloides & 156 \\
Oesophagostomum spp. & 156 \\
\hline
\end{tabular}

Larvas do gênero Haemonchus spp, Cooperia spp., Trychostrongylus spp., Oesophagostomum spp., Bunostomum spp. e Strongyloides foram encontradas parasitando os bezerros e identificadas em sua forma de primeiro estágio, conforme metodologia proposta por Sprent (1946), Hansen e Shivnani (1956), Rodrigues e Honer (1985) e Reinecke e Reinecke (1993).
Repossi Júnior et al. (2006) observaram que os gêneros de larvas identificadas com maior prevalência foram Cooperia spp. e Hamonchus spp., em bezerros provenientes de propriedades leiteiras do município de Alegre, Espírito Santo, corroborando com os resultados do presente estudo.

Duarte, Gomes e Sant'Anna (1982), em estudo realizado com bezerros do município de Cantagalo, 
estado do Rio de Janeiro, também observaram maior prevalência destes dois gêneros de nematóides em relação a outros helmintos gastrintestinais de bovinos. Landim et al. (2001), em estudo realizado na região nordeste de São Paulo, também observaram maior parasitismo causado por Cooperia spp. e Haemunchus spp. em bovinos.

Guimarães et al. (2000) observaram além destes dois gêneros, a presença de Trichostrongylus spp e Oesophagostomum spp. em estudo com gado Zebu, no estado de Minas Gerais, sendo semelhante ao resultado observado no presente estudo.

Duarte Gomes e Sant' Anna (1982) demonstraram que a prevalência de Trichostrongylus spp. foi baixa em relação aos gêneros Cooperia spp. e Haemonchus spp. Resultado similar foi observado por Borges et al. (2001), em estudo realizado com bezerros da região de Jaboticabal, São Paulo.

Lima (1981) observou um número baixo de ovos de Trichostrongylus spp. em amostras de fezes de bezerros do município de Valença, estado do Rio de Janeiro, diferindo dos resultados obtidos no presente estudo.

Contudo, a elevada prevalência observada para este gênero, no presente estudo, pode estar relacionada à influência do microclima mais ameno observado na região estudada, pois temperaturas elevadas influenciam negativamente os estados pré-infectantes dessas espécies de nematóides (ROBERTS; O'SULLIVAN; RIEK, 1952; LEE; ARMOUR; ROSS, 1960).

Em relação ao gênero Oesophagostomum spp., Duarte Gomes e Sant'Anna. (1982) observaram prevalência de aproximadamente $22 \%$ dos bezerros, no estado do Rio de Janeiro, valor este um pouco abaixo do observado no presente estudo. Porém, Oliveira e Matsumoto (1985) e Pimentel Neto e Fonseca (2002) observaram prevalência acima de $80 \%$ para este gênero no estado de São Paulo e Rio de Janeiro, respectivamente.

O gênero Bunostomum spp. apresentou baixa ocorrência, sendo observado resultados similares aos estudos de Borges et al. (2001), Oliveira e Matsumoto (1985) e Pimentel Neto e Fonseca (2002). Em relação ao gênero Strongyloides, Duarte Gomes e Sant'Anna (1982) observaram prevalência de aproximadamente $31 \%$, próximo ao valor observado no presente estudo.

Em relação às médias de $\mathrm{L}_{1}$ pode-se observar que, a média de larvas de Cooperia spp. foi bem mais elevada que os demais gêneros identificados, sendo 1,7 vezes maior que o gênero Haemonchus spp. Esse resultado pode estar associado à intensidade de parasitas nos bezerros examinados, como observado por Guimarães et al. (1983) e Pimentel Neto e Fonseca (2002), demonstrando que a carga parasitária por Cooperia spp. foi bem acima da observada por Haemonchus spp.

Apesar das fêmeas do gênero Haemonchus spp. possuírem um maior potencial de postura diária (5000-10.000) em relação as fêmeas do gênero Cooperia spp.(100-200) (UENO; GONÇALVES, 1998), ambos os gêneros causam infecções em praticamente $100 \%$ dos animais da região Sudeste (HONER; VIEIRA-BRESSAN, 1991).

Segundo Cezar, Catto e Bianchim (2008), o controle das helmintoses torna-se mais eficiente quando existem conhecimentos epidemiológicos básicos, destacando-se características regionais ou mesmo específicas do local e do tipo de sistema produtivo adotado.

Em relação à técnica utilizada no presente trabalho, como protocolo de diagnóstico das helmintoses em bovinos, pode-se destacar, dentre suas vantagens, a maior rapidez na obtenção dos resultados (em até 24 horas) dos gêneros de nematóides gastrintestinais; quando comparada a técnicas clássicas como as descritas por Roberts e O'Sulivan (1950), Whitlock (1959) e Rodrigues e Honer (1985). Desta forma, através da técnica preconizada pode-se se diagnosticar seis gêneros de nematóides presentes nas amostras desses bezerros, sendo identificados com maior freqüência os gêneros Cooperia spp., Haemonchus spp. e Trichostrongylus spp. 


\section{Agradecimentos}

Os autores agradecem ao professor emérito Dr. Richard Karl Reinecke (in memorian) da Universidade de Petrória, África do Sul, por todo incentivo, apoio e orientações no desenvolvimento da pesquisa e ao professor Dr. Adivaldo Henrique da Fonseca, pela revisão do artigo. Agradecem também ao apoio dado pelo Dr. Carlos Vildoso Maldonado, a toda a equipe da EMATER-RJ de Paty do Alferes, juntamente com todos os criadores da região que gentilmente nos cederam os animais, permitindo desta forma a realização deste trabalho.

\section{Referências}

ABIDU-FIGUEIREDO, M.; CARVALHO-DE-SOUZA, B.; MAIO, F. G.; BARBOSA, C. G.; REINECKE, R. K. Comparação entre técnicas coproparasitológicas para a contagem de ovos de nematóides gastrointestinais de búfalos. Revista Científica Eletrônica de Medicina Veterinária, Garça, v. 3, n. 5, p. 1-8, 2005. Disponível em: <http://www.revista.inf.br/veterinaria05/artigos/ artigo07.pdf>. Acesso em: 29 jul. 2009.

AMARANTE, A. F. Controle integrado de helmintos de bovinos e ovinos. Revista Brasileira de Parasitologia Veterinária, Jaboticabal, v. 13, n. 1, p. 68-71, 2004.

AYRES, M. BioEstat 2.0 - Aplicações estatísticas nas áreas das Ciências Biológicas e Médicas. Sociedade Tefé, Belém: Civil Mamirauá, 2000. 272 p.

BIANCHIN, I. Epidemiologia e controle de helmintos gastrointestinais em bezerros a partir da desmama, em pastagem melhorada, em clima tropical do Brasil. 1991. Tese. (Doutorado em Parasitologia Veterinária) Universidade Federal Rural do Rio de Janeiro, Rio de Janeiro.

BIANCHIN, I.; HONER, M. R.; NUNES, S. G.; NASCIMENTO, Y. A.; CURVO, J. B. E.; COSTA, F. P. Epidemiologia dos nematódeos gastrintestinais em bovinos de corte nos cerrados e o controle estratégico no Brasil. Campo Grande: EMBRAPA, CNPGC, 1993. 120 p. (Circular Técnica, 24).

BORGES, F. A.; SILVEIRA, D. M.; GRAMINHA, N. E. B.; CASTAGNOLLI, K. C.; SOARES, V. E.; NASCIMENTO, A. A.; COSTA, A. J. Fauna helmintológica de bovinos da região de Jaboticabal, Estado de São Paulo, Brasil. Semina: Ciência Agrárias, Londrina, v. 22, n. 1, p. 49-53, 2001.
CEZAR, A. S.; CATTO, J. B.; BIANCHIM, I. Controle alternativo de nematódeos gastrintestinais dos ruminantes: Atualidade e perspectivas. Ciência Rural, Santa Maria, v. 38, n. 7, p. 2083-2091, 2008.

DUARTE, M. J. F.; GOMES, P. A. C.; SANT'ANNA, D. B. Prevalência e intensidade de infecção helmíntica em bezerros de Cantagalo, RJ, Brasil. Pesquisa Agropecuária Brasileira, Brasília, v. 17, n. 10, p. 1521-1524, 1982.

EYSKER, M.; PLOEGER, H. W. Value of present diagnostic methods for gastrointestinal nematode infections in ruminants. Parasitology, Cambridge, v. 120, n. 7, p. 109-119, 2000.

FORBES, A. B.; CUTLER, K. L.; RICE, B. J. Subclinical parasitism in spring-born, beef suckler calves: epidemiology and impact on growth performance during the first grazing season. Veterinary Parasitology, Amsterdam, v. 104, n. 4, p. 339-344, 2002.

GUIMARÃES, M. P.; LIMA, W. S.; LEITE, A. C. R.; COSTA, J. O. Gastrointestinal nematode infection in beef cattle from the Savannah Region ("Cerrado") of Brazil. Arquivo Brasileiro de Medicina Veterinária e Zootecnia, Belo Horizonte, v. 35, n. 6, p. 845-851, 1983.

GUIMARÃES, M. P.; RIBEIRO, M. F. B.; FACURIFILHO, E. J.; LIMA, W. S. Strategic control of gastrointestinal nematodes in dairy calves in florestal, Minas Gerais, Brazil. Veterinary Research Communications, Amsterdan, v. 24, n. 1, p. 31-38, 2000.

HANSEN, M. F.; SHIVNANI, G. Comparative morphology of infective nematode larvae of Kansas beef cattle and its use in estimating incidence of Nematodiasis in cattle. Transactions of the American Microscopical Society, Columbus, v. 75, n. 1, p. 91-102, 1956.

HONER, M. R.; VIEIRA-BRESSAN, M. C. Nematóides de bovinos do Brasil: o estado da pesquisa. Revista Brasileira de Parasitologia Veterinária, Jaboticabal, v. 1, n. 1, p. 67-79, 1991.

LANDIM, V. J. C.; COSTA, A. J.; COSTA, G. H. N.; ROCHA, U. F.; BARBOSA, O. F.; MORAES, F. R. Parasitic nematodes in weaned calves from the northeast region of São Paulo state, Brazil. Ars Veterinária, Jaboticabal, v. 17, n. 1, p. 42-50, 2001.

LEE, R. P.; ARMOUR, J.; ROSS, J.G. The seasonal variations of strongyle in Nigerian zebu cattle. British Veterinary Journal, London, v. 116, n. 1, p. 34-46, 1960.

LIMA, M. M. Helmintiases gastrintestinais em bovinos de raça leiteira. 1981. Dissertação (Mestrado em Parasitologia Veterinária) - Universidade Federal Rural do Rio de Janeiro, Rio de Janeiro. 
OLIVEIRA, G. P.; MATSUMOTO, T. Prevalência e intensidade de infecção por helmintos em bovinos da bacia leiteira de São Carlos, São Paulo. Pesquisa Agropecuária Brasileira, Brasília, v. 20, n. 12, p. 14151418, 1985.

PIMENTEL NETO, M.; FONSECA, A. H. Epidemiologia das helmintoses pulmonares e gastrintestinais de bezerros em região de baixada do Estado do Rio de Janeiro. Pesquisa Veterinária Brasileira, Rio de Janeiro, v. 22, n. 4, p. 148-152, 2002.

REINECKE, R. K.; FIGUEIREDO, M. A.; MATTOS JUNIOR, D. G. Um método simples para o diagnóstico dos nematóides gastrointestinais mais comuns em bezerros. In: CONGRESSO DE MEDICINA VETERINÁRIA, 22., 1992, Curitiba. Anais...Curitiba, 1992. p.188.

REINECKE, R. K.; FONSECA; A. H. First stage larvae per $15 \mathrm{ml}\left(\mathrm{L}_{1} .15 \mathrm{ml}^{-1}\right)$ calf faeces for the diagnosis of nematode parasites "ante mortem". Pesquisa Veterinária Brasileira, Rio de Janeiro, v. 1/2, n. 12, p. 43-44, 1992.

REINECKE, R. K.; REINECKE, N. D. Identification of first estage larvae $\mathrm{L}_{1}$ of bovine nematodes. Pesquisa Veterinária Brasileira, Rio de Janeiro, v. 1, n. 14, p. 1-4. 1993.

REPOSSI JUNIOR, P. F.; BARCELLOS, M. P.; TRIVILIN, L. O.; MARTINS, I. V. F.; SILVA, P. C. A. R. Prevalência e controle das parasitoses gastrintestinais em bezerros de propriedades leiteiras no município de Alegre, Espírito Santo. Revista Brasileira de Parasitologia Veterinária, Jaboticabal, v. 15, n. 4, p. 147-150. 2006.
ROBERTS, F. H. S.; O'SULLIVAN, P. J.; RIEK, R. F. The epidemiology of parasitic gastro-enteritis of cattle. Australian Journal of Agricultural Research, Victoria, v. 3, n. 2, p. 187-226, 1952.

ROBERTS, F. H. S.; O'SULLIVAN, P. S. Methods for egg counts and larval culture for strongyle infecting the gastrointestinal tract of cattle. Australian Journal of Agricultural Research, Victoria, v. 1, n. 1, p. 99-102, 1950.

RODRIGUES, M. L. A.; HONER, M. H. Obtenção e identificação de larvas de primeiro estágio $\left(\mathrm{L}_{1}\right)$ de nematóides gastrointestinais de bovinos: Modificação da técnica de Whitlock (1959). Pesquisa Veterinária Brasileira, Rio de Janeiro, v. 5, n. 1, p. 1-3, 1985.

SPRENT, J. F. A. Studies on the lyfe history of Bunostomum phlebotomum (Railliet, 1900), a hookworm parasite of cattle. Parasitology, Cambridge, v. 37, p. 192 201, 1946.

UENO, H.; GONÇALVES, P. C. Manualpara diagnóstico das Helmintoses de ruminantes. 4. ed. Tóquio: JICA, $1998.143 \mathrm{p}$.

WALLER, P. J. International approaches to the concept of integrated control of nematode parasites of livestock. International Journal for Parasitology, Oxford, v. 29, n. 1, p. 155-164, 1999.

WHITLOCK, H. V. The recovery and identification of the first stage larvae of sheep nematodes. Australian Veterinary Journal, St-Leonardes, v. 35, n. 7, p. 310-316, 1959. 